\title{
QUEEN'S
UNIVERSITY
BELFAST
}

\section{The illusion of replacement in research into the development of thinking biases: the case of the conjunction fallacy}

Morsanyi, K., Chiesi, F., Primi, C., \& Szucs, D. (2016). The illusion of replacement in research into the development of thinking biases: the case of the conjunction fallacy. Journal of Cognitive Psychology. https://doi.org/10.1080/20445911.2016.1256294

Published in:

Journal of Cognitive Psychology

Document Version:

Peer reviewed version

Queen's University Belfast - Research Portal:

Link to publication record in Queen's University Belfast Research Portal

Publisher rights

(C) 2016 Informa UK Limited, trading as Taylor \& Francis Group

This is an Accepted Manuscript of an article published by Taylor \& Francis in Journal of Cognitive Psychology on 09 Nov 2016, available online: http://www.tandfonline.com/doi/full/10.1080/20445911.2016.1256294

\section{General rights}

Copyright for the publications made accessible via the Queen's University Belfast Research Portal is retained by the author(s) and / or other copyright owners and it is a condition of accessing these publications that users recognise and abide by the legal requirements associated with these rights.

Take down policy

The Research Portal is Queen's institutional repository that provides access to Queen's research output. Every effort has been made to ensure that content in the Research Portal does not infringe any person's rights, or applicable UK laws. If you discover content in the Research Portal that you believe breaches copyright or violates any law, please contact openaccess@qub.ac.uk. 


\author{
Morsanyi, K., Chiesi, F., Primi, C., \& Szücs, D. (2016). The illusion of replacement in \\ research into the development of thinking biases: the case of the conjunction fallacy. \\ Journal of Cognitive Psychology (in press)
}

DOI:10.1080/20445911.2016.1256294

\title{
ABSTRACT
}

The conjunction fallacy is a violation of a very basic rule of probability. Interestingly, although committing the fallacy seems irrational, adults are no less susceptible to the fallacy than young children. In Experiment 1, by employing tasks where the conjunctive response option involved two non-representative items, we found a large reduction in fallacy rates as compared to traditional conjunction fallacy problems. Nevertheless, fallacy rates remained relatively high in both adolescents and adults, although adults showed more consistency in their normative responses. In Experiment 2, we demonstrated that children's relatively good performance on the task was not the consequence of their missing knowledge of social stereotypes. Additionally, children were more strongly affected by explicitly presented frequency information than adults. Indeed, adults only took frequency information into account when frequencies were made relevant by a training in probabilistic reasoning. Overall, the results suggest that whereas the potential for normative reasoning increases with development, this potential is often overshadowed by a pervasive tendency in adolescence and adulthood to rely on contextual information, knowledge, and beliefs, even when conflicting information is available. By contrast, children are more strongly influenced by explicitly presented information than relevant knowledge cued by the tasks.

KEYWORDS: Cognitive development; conjunction fallacy; contextualisation; frequency information; inverted U-shaped pattern; rationality

Researchers of the development of judgment and reasoning generally assume that adults are characterised by an increased tendency for normative and rule-based reasoning as compared to children (e.g., Stanovich, Toplak, \& West, 2008). At the same time, it is well-documented that adults often exhibit systematic deviations from normative rules, and they are susceptible to biases and the inappropriate use of mental shortcuts (see, e.g., Gilovich, Griffin, \& Kahneman, 2002). Given that adults often exhibit suboptimal performance on reasoning tasks, the general assumption is that children's performance on these problems must be even worse (or they might simply show random performance-cf. Stanovich, West, \& Toplak, 2011). In fact, children's performance on these tasks is rarely investigated, and we know relatively little about their judgment and reasoning skills, and the underlying factors.

The aim of the present paper is to investigate developmental patterns from childhood to adulthood, as well as potential explanations for the observed developmental changes, using problems that are known to elicit the conjunction fallacy. The conjunction fallacy is a violation of a very basic rule of probability-that the probability of two events happening together (i.e., A\&B) cannot be higher than the probability of either constituent event happening (e.g., A). People who commit the conjunction fallacy assign a higher probability to a conjunction than to one, or both, of its constituents. In the most famous demonstration of the fallacy (Tversky \& Kahneman, 1983), participants were presented with a description of Linda, a 31-year-old, smart, outspoken woman who was a philosophy major, concerned with discrimination and social justice, and a participant in antinuclear demonstrations. Participants were then asked to judge a number of statements about Linda according to how likely they were. When assessing the relative likelihood of the 
statements, the vast majority of participants ranked the statement "Linda is a bank teller and is active in the feminist movement" above the statement "Linda is a bank teller"-thus, committing the fallacy.

Whereas much research has addressed the question of why fallacy rates are high amongst educated adults (Adler, 1984; Politzer \& Noveck, 1991; Tversky \& Kahneman, 1982), much fewer studies investigated developmental changes in fallacy rates. Although one could expect that fallacy rates should be especially high in the case of children (as they can be expected to be less familiar with the conjunction rule), this does not seem to be a case. Davidson (1995) and Morsanyi and Handley (2008) used child-friendly versions of the original "Linda problem", and they found that conjunction fallacy rates increased during the primary school years (between the ages of 5 and 11). Morsanyi and Handley (2013) extended this work to adolescents and young adults, and they reported an inverted U-shaped developmental pattern from childhood to adulthood, where fallacy rates increased during childhood, were at ceiling in adolescence, and then decreased again between adolescence and young adulthood. Interestingly, fallacy rates were equal between the child and adult samples.

Inverted U-shaped developmental patterns are typically attributed to an interaction between two distinct cognitive processes that act in opposition, and that emerge at different points during development. In the case of the conjunction fallacy, the two opposing factors that have been proposed are the influence of the representativeness of the response options, and adherence to the conjunction rule (Tversky \& Kahneman, 1983). The inverted U-shaped developmental pattern might be explained by assuming an initial increase in representativeness-based responses, which could be a consequence of increasing familiarity with the relevant stereotypes with age (e.g., Toplak, West, \& Stanovich, 2014). Then, once participants learn the conjunction rule, fallacy rates decrease.

Indeed, some authors (De Neys \& Vanderputte, 2011; Stanovich et al., 2008, 2011; Toplak et al., 2014) have argued that the counterintuitive developmental patterns in childhood (i.e., an increase in fallacy rates) were simply the side-effects of using materials that were inappropriate for young children. Specifically, given that children were less familiar with the stereotypes evoked by the descriptions in the problems, they were also less influenced by the representativeness of the descriptions (as, for them, these descriptions did not seem representative to a particular group in the first place). In fact, although the problems included in Davidson (1995) and Morsanyi and Handley (2008) were designed for child participants, these authors did not check if the participants in their studies were actually familiar with the stereotypes evoked by the problems. Thus, one aim of the current work was to revisit this question by investigating conjunction fallacy rates in children, adolescents, and adults, making sure that only those participants who were familiar with the relevant stereotypes were included in the comparisons.

Regarding the effect of the conjunction rule on responding, there is evidence that this increases with age. In particular, two studies, Chiesi, Gronchi, and Primi (2008) and Fisk and Slattery $(\underline{2005})$, investigated familiarity with the conjunction rule in the case of children and adults using materials that did not cue pre-existing knowledge about social stereotypes. Chiesi et al. (2008) presented their participants with pictorial materials of flowers with or without a bee on them (A\&B and A\&notB). Chiesi et al. (2008) included participants from three age groups: 7-year-olds, 10year-olds, and young adults. The participants were presented with different scenarios where the proportion of $A \& B$ and $A \& n o t B$ items was systematically manipulated. For each scenario participants had to choose from three response options: $A \& B, A$, or "both response options are equally likely". Whereas the youngest children were not influenced by the relative frequencies of $A \& B$ and $A \& n o t B$, older participants were strongly affected by frequency information, and this effect was the strongest in the case of adults (see also Chiesi, Primi, \& Morsanyi, 2011; De Neys \& Vanderputte, 2011 for evidence for increasing sensitivity to numerical and probability information with development). At the same time, the results suggested that both older children and adults interpreted the question as regarding a comparison between $A \& B$ and $A \& n o t B$ (instead of $A \& B$ vs. A) which is in line with a particular pragmatic explanation of the conjunction fallacy (e.g., Adler, 1984; Politzer \& Noveck, 1991). Moreover, the fact that this kind of 
interpretation was more prevalent in older participants is in line with other studies which have shown that sensitivity to pragmatic cues increases with development (see Feeney, Scrafton, Duckworth, \& Handley, 2004; Noveck, 2001). Thus, these results not only suggested an increased sensitivity to probability information in older participants, but also an increased tendency to rely on pragmatic inferences.

Fisk and Slattery (2005) used a different version of the task where, similar to Chiesi et al. (2008), frequency information was explicitly provided, and pre-existing knowledge was not cued by the problem. Moreover, the materials were linguistically unambiguous (i.e., they did not allow for participants to interpret $A$ as $A \& n o t B)$. There were three groups of participants: 5-year-olds, 9year-olds, and young adults. The task measured participants' tendency to commit the conjunction fallacy when the conjunctive option was paired with a single non-representative event vs. when it was paired with a single representative event. Children from the two age groups showed virtually identical performance: about $60 \%$ fallacy rate in the case of a non-representative single event, and $40 \%$ fallacy rate in the case of a representative single event. For adults, fallacy rates were significantly lower: $29 \%$ and $9 \%$, respectively. That is, based on these results, it could be concluded that about $29 \%$ of adults did not rely on the conjunction rule when a linguistically unambiguous task was used to measure the conjunction fallacy and probability information was presented explicitly. It is also clear that fallacy rates were much higher in the case of children, although it is possible that some of these errors stemmed from the relative complexity of the task. Nevertheless, overall both Chiesi et al.'s (2008) and Fisk and Slattery's (2005) studies suggest that adults are better able to apply the conjunction rule than children.

Although unfamiliarity with relevant social stereotypes could potentially explain the counterintuitive developmental trajectory of the conjunction fallacy, we present an alternative explanation. Specifically, we question a general assumption about reasoning development-that cognitive maturation involves a move away from simple heuristic processing towards effortful, normative reasoning. We are not the first developmental researchers to raise this issue. For example, Reyna and Ellis (1994) referred to the idea that reasoning development involves a gradual shift from predominantly heuristic to predominantly effortful reasoning as the "illusion of replacement". In line with some earlier work, in the current paper we will argue that whereas the potential for normative reasoning increases with development, heuristic processing also develops (see, e.g., Morsanyi \& Handley, 2008; Reyna \& Brainerd, 2011). That is, "simple heuristics" might not be as simple as they appear, and, in fact, reasoning development incorporates both an increased ability to reason on the basis of normative rules, and to use shortcuts.

With regard to the conjunction fallacy, Morsanyi and Handley (2008) not only showed an increase in fallacy rates with age in the case of children, but they also showed that fallacy rates were positively related to children's cognitive capacity (after controlling for the effect of age). Additionally, Morsanyi, Handley, and Evans (2010) found that adolescents with autism (a population characterised by a reduced tendency to spontaneously contextualise presented information, $\stackrel{1}{ }$ and a tendency to focus on verbatim details-see, e.g., Happé \& Frith, 2006) were less susceptible to the fallacy than typically developing adolescents, although they were equally able to recognise the stereotypes cued by the descriptions. Additionally, in the case of adolescents with autism, the tendency to commit the fallacy was positively related to participants' verbal ability.

These findings suggest that at least some reasoning heuristics (such as the conjunction fallacy, for example) can be considered as developmental achievements, and these types of responses appear to require cognitive effort in the case of young children, as well as adolescents with autism. Morsanyi and Handley (2013) interpreted these findings as evidence that the ability to utilise pre-existing knowledge in the context of a reasoning task (a tendency that pervades adult reasoning) develops in children slowly and gradually. Thus, it is not a lack of relevant knowledge that prevents children from relying on context-based heuristics, but a difficulty with a spontaneous, flexible, and effortless application of this knowledge. 
Taken together, existing studies have provided some intriguing findings regarding developmental changes in the conjunction fallacy, and competing explanations for these developmental patterns have been offered by different theorists. In particular, it is of interest why in studies using "Linda problem"-type tasks susceptibility to the conjunction fallacy increases during childhood. Specifically, we were interested in whether this counterintuitive developmental pattern could be eliminated by ensuring that participants who are unfamiliar with the relevant social stereotypes are excluded from the developmental comparisons.

Another aim of our paper was to demonstrate that, in contrast with children, adults show an excessive sensitivity to context. Indeed, this tendency is so pervasive that it has been termed the "fundamental computational bias" of human cognition (Stanovich, 2003). Although the majority of adult participants are familiar with the conjunction rule (e.g., Fisk \& Slattery, 2005), they tend not to apply this knowledge in the context of typical conjunction fallacy tasks. Indeed, manipulations that are aimed at eliminating the fallacy, including both pragmatic modifications (Ahn \& Bailenson, 1996; Gigerenzer, 1996; Hertwig, Benz, \& Krauss, 2008; Tentori, Bonini, \& Osherson, 2004) and the inclusion of frequency information (e.g., Tentori et al., 2004; Wedell \& Moro, 2008), usually have limited impact on fallacy rates. In line with our account that assumes reduced sensitivity to contextual cues in children, in the current paper, we test the counterintuitive prediction that children might be more sensitive than adults to some experimental manipulations that are aimed at reducing fallacy rates. Specifically, whereas it can be expected that children would be less sensitive to pragmatic cues (Feeney et al., 2004; Noveck, 2001), they might show relatively strong sensitivity to explicitly presented frequency information. By contrast, adolescents might be particularly insensitive to such manipulations, as it can be expected that they have already developed the ability to spontaneously contextualise information, but they have more limited knowledge of normative rules than adults, and reduced ability to apply these rules.

In order to investigate these predictions, in Experiment 1, together with traditional conjunction fallacy problems, we included problems with two non-representative conjuncts, in order to make the conjunctive response option less attractive. We expected that this manipulation would encourage participants to rely on the conjunction rule. In particular, by making the conjunctive option less attractive, we hoped to prompt a more careful consideration of the problems. We were also interested in whether this manipulation would affect adolescents and adults differently.

Experiment 2 included a developmental comparison between children and adults. In this study, in addition to a traditional conjunction fallacy task, we also used a modified task, where frequency information was provided together with the response options. It was emphasised that a random selection process was employed, in order to highlight the relevance of frequency information. Additionally, some of our adult participants were provided with a training in probabilistic reasoning, to further encourage reliance on numerical/probabilistic information. Whereas on the basis of traditional approaches to cognitive development it could be expected that adults should be more sensitive to frequency information, we expected that children would be at least as much (or even more strongly) affected by frequency information as adults, given their reduced sensitivity to contextual information and stronger focus on explicitly presented details.

In summary, the purpose of the following studies was twofold. First, we aimed to investigate developmental changes in the conjunction fallacy (using versions of the task which were comparable to the original Linda problem). In both experiments, we included the representative item alone as a response option, and we only included responses from participants who marked this as the most likely. This way, we were able to make comparisons between age groups with regard to their ability to recognise relevant stereotypes, and to use these as the basis of their likelihood ratings. Additionally, this made it possible for us to control the potential effects of stereotype-familiarity on developmental patterns. The second aim was to test a particular developmental account of the conjunction fallacy. According to this account, although the potential for normative responding increases with development, this potential often remains unexploited, even in the case of educated adults (see Experiments 1 and 2). We will argue that this is because adults place a great emphasis on the context in which different pieces of information are presented, at the expense of considering various pieces of information at their 
face value. At the same time, contextual information can be helpful in highlighting the relevance of certain details that are important for normative responding (see Experiment 2). By contrast, children are more focused on explicitly presented details and, as a result, they might reason at the same level or even more "normatively" than adults in some cases (Experiment 2).

\section{Experiment 1}

Our first experiment investigated developmental changes in susceptibility to the conjunction fallacy between early adolescence and adulthood. Previous studies (Klaczynski, 2001a; Morsanyi \& Handley, 2013) reported no age-related change in fallacy rates in adolescence, and, at the same time, they showed that fallacy rates were at (or very close to) ceiling. However, Morsanyi and Handley (2013) found a reduction in fallacy rates in the case of young adults (as compared to adolescents). Thus, one aim of the study was to confirm these developmental patterns in a new sample. Another aim was to explore the effect of an experimental manipulation that was aimed at reducing fallacy rates. Specifically, beside traditional conjunction fallacy problems (where the conjunctive response option included a representative and a nonrepresentative item), we also administered a different version of the problems, which included the conjunction of two non-representative items-see Table 1. By making the conjunctive option less attractive, we hoped to encourage a more careful consideration of the response options, which could potentially increase reliance on the conjunction rule.

Table 1. Examples of traditional (including the conjunction of a representative and a nonrepresentative item) and non-representative (including the conjunction of two non-representative items) versions of a problem.

\begin{tabular}{||l||c||}
\hline Traditional version & Non-representative version \\
\hline \hline $\begin{array}{l}\text { Brian has a studio, where he works alone. He is a very creative man, and he likes to experiment } \\
\text { with colours. He takes his work to exhibitions, and sells some of them too. Mark the following } \\
\text { statements with number } 1 \text { to } 4 \text { according to how likely they are. (1: most likely, } 4 \text { : least likely) }\end{array}$ \\
\hline \hline Brian is an aerobics instructor. & Brian is an aerobics instructor. \\
\hline \hline Brian is a painter. & Brian is a painter. \\
\hline Barian is a painter and an & Brian is an aerobics instructor and an \\
\hline \hline Brian is an accountant. & Brian is an accountant. \\
\hline \hline
\end{tabular}

Note: Traditional and non-representative versions of problems with the same content were administered to different groups of participants.

Note that even in the case of tasks with non-representative conjuncts, it is likely that participants still consider the representativeness of the conjunctive option, possibly by using some kind of averaging strategy (see, e.g., Aczel, Szollosi, \& Bago, 2016; Fantino, Kulik, Stolarez-Fantino, \& Wright, 1997; Fisk \& Pidgeon, 1996; Nilsson, Winman, Juslin, \& Hansson, 2009). Nevertheless, given the implausibility of the response options, it is likely that participants will search for other possible cues for weighing the response options, which could make the relevance of the conjunction rule more salient. We were also interested in whether this manipulation would affect 
adults and adolescents equally. In particular, adults might be more sensitive to this manipulation, as they have higher cognitive capacity, and they are more likely to be familiar with the conjunction rule than adolescents.

\section{Method}

\section{Participants}

Eighty-six participants were involved in this study. Fifty-eight students were recruited from years 7 to 12 of two secondary schools in the South-East and South-West of England. In order to explore developmental changes in the conjunction fallacy during adolescence, we created two age groups: early adolescents (years $7-9$ of secondary school; $n=29 ; 13$ boys; mean age: 12 years 3 months), and mid-to-late adolescents (years 10-12; $n=29 ; 12$ boys; mean age: 15 years 2 months). Additionally, 28 adult participants (mean age: 30 years 4 months; 11 males), recruited through a paid participant pool in the South-East of England, took part in the experiment.

\section{Materials}

\section{Conjunction fallacy problems}

We used four conjunction fallacy problems (based on Morsanyi et al., 2010; see Appendix). In each task, participants had to rate four statements according to how likely they thought that the statements were true. One statement was representative (based on the description), two statements were non-representative, and the fourth statement was either a conjunction of a representative and a non-representative statement (i.e., in traditional conjunction fallacy problems) or a conjunction of two non-representative statements-see Table 1 . In order to minimise content effects, we designed two sets of problems. Half of the participants in each year group were administered Set 1, and the other half were administered Set 2 . The two sets of problems were designed in a way so that participants in one group solved the traditional version, and the other half of participants solved the modified version of the same problem (i.e., a problem which had exactly the same content, but included the conjunction of two nonrepresentative items), and each participant solved two problems of each type. In the case of the traditional problems, the measure of the conjunction fallacy was whether participants judged the probability of the conjunction of a representative and a non-representative statement as more likely to be true than the non-representative conjunct alone. In the tasks with non-representative conjuncts, the dependent measure was whether participants judged the conjunction of two nonrepresentative statements as more likely to be true than at least one of the conjuncts alone. Thus, we computed average fallacy rates taking into consideration both possible ways of committing the fallacy (i.e., if a person rated the conjunction as more likely than at least one of the conjuncts, then they committed the fallacy). Note that in the case of traditional problems there was only one possible way of committing the fallacy, given that we only included those participants in our analyses who marked the representative single event as the most likely. That is, theoretically, the likelihood of committing the fallacy in the case of non-traditional problems was twice as high as in the case of traditional problems. In summary, participants were administered two problems of each type. Each task was scored as 0 (did not commit the fallacy) or 1 (committed the fallacy), and we computed fallacy rates based on the average of these two scores. Thus, for both types of problem, there were three possible values: $0,0.5$, and 1.

\section{Procedure}

The conjunction fallacy problems were administered together with some other tasks (not reported here) as part of a group session. ${ }^{2}$ The different types of problems were presented in a booklet, together with the instructions. The problems were presented in a quasi-random order (with the 
constraint that two problems of the same type-for example, two conjunction fallacy problemswere not presented consecutively). The experimenter read out the instructions, and the participants worked through the problems individually, at their own pace. The testing session took about 20 min altogether.

\section{Results}

In our analyses, we computed average conjunction fallacy rates only on the basis of those problems where participants marked the representative option as the most likely. We assumed that participants who identified the representative option as the most likely followed our instructions regarding how to mark the options, and they were also affected by the representativeness of the description (i.e., they did not avoid the fallacy in the case of a traditional problem simply because the item that they considered to be the most representative was not included in the conjunction). Early adolescents marked the representative option as the most likely in $88 \%$ of the cases, late adolescents did this in $92 \%$ of the cases, and adult participants did this in $95 \%$ of the cases. A chi-square test indicated that the difference between age groups was not significant $(p=.18)$.

Our analysis aimed at establishing whether participants were sensitive to our manipulation of the representativeness of the conjunctive response option (i.e., whether participants were less likely to commit the fallacy when the task included the conjunction of two non-representative events, as opposed to when it included the conjunction of a representative and a non-representative event). We also contrasted fallacy rates across the three age groups in order to investigate potential developmental changes in responding (see Figure 1).

Figure 1. Fallacy rates across problem types and age groups. Error bars represent $95 \%$ confidence interval of the means.

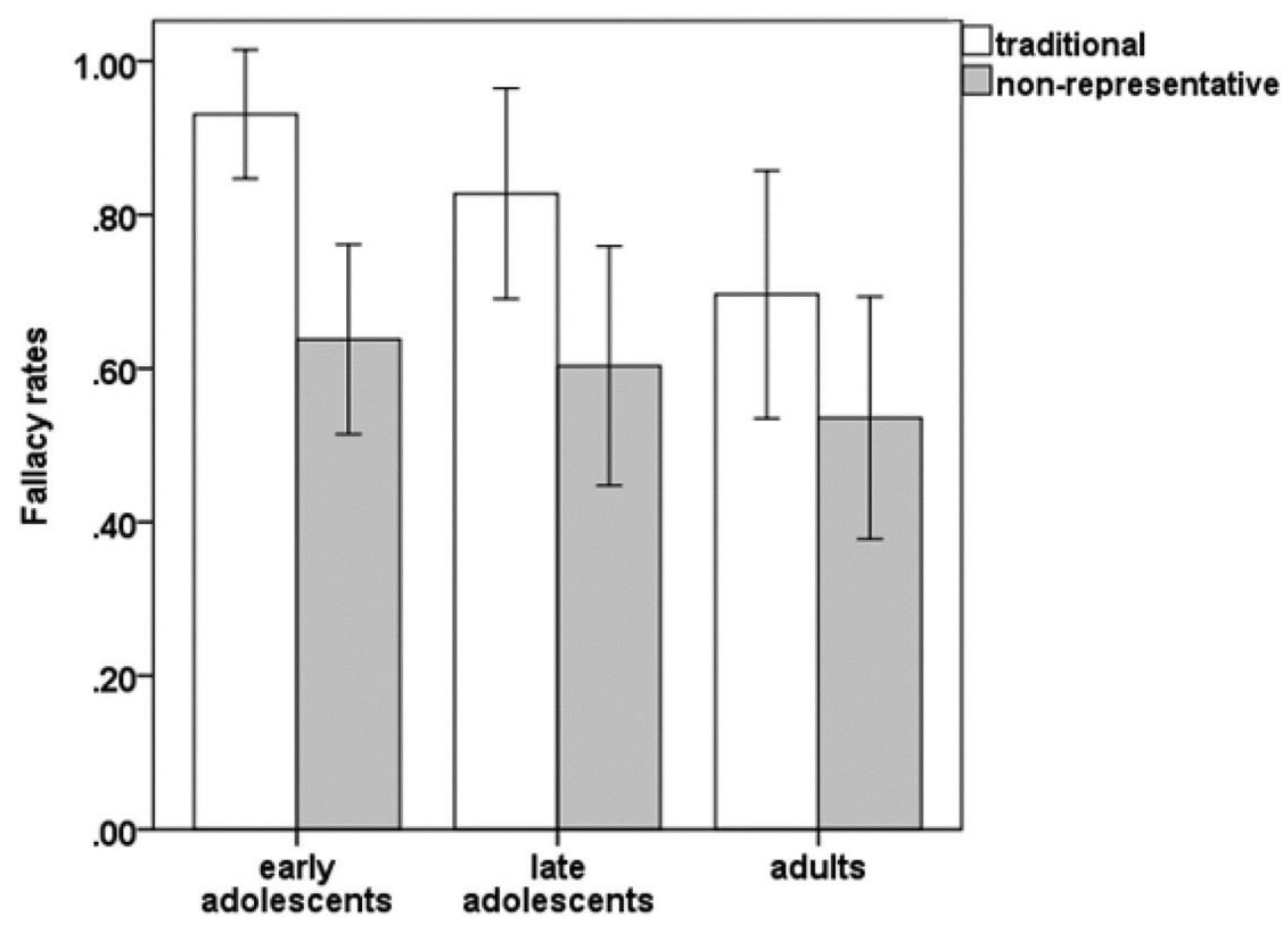


A $2 \times 3$ mixed ANOVA with task version (traditional/task with non-representative conjuncts) as a within-subjects factor, and age group (early adolescent/late adolescent/adult) as a $\eta_{\mathrm{p}}^{2}$ tweensubjects factor indicated a significant effect of task version $\left.\eta_{\mathrm{p}}^{2} 1,83\right)=19.10, p<.001, \quad=.19$ ), and a marginal effect of age group $F(2,83)=2.77, p=.069, \quad=.06$ ), but no interaction between the two factors $(p=.58)$. That is, participants were more susceptible to the fallacy when the conjunction included a representative conjunct $(M=.82, S D=.35)$, as compared to when both conjuncts were non-representative $(M=.59, S D=.38)$.

We have also computed the proportion of participants who consistently avoided the fallacy in the case of both the traditional version of the task, and the task with non-representative conjuncts (see Figure 2). We included this additional analysis, as a consistent avoidance of the fallacy could give a stronger indication that participants were relying on normative considerations, instead of avoiding the fallacy because they found the conjunctive option particularly unrepresentative. A $2 \times 3$ mixed ANOVA with task version (traditional/task with nonrepresentative conjuncts) as a within-subjects factor, and age group (early adolescent/late adolescent/adult) as a between-subjects factor indicated an effect of age group $(F(2,83)=3.47$, $p=.036, \eta_{\mathrm{p}=.08}^{2}$ ), but no effect of task version $(p=.151)$, and no interaction between the two factors $(p=.961)$. A post-hoc comparison using Tukey's $b$ test indicated that the proportion of participants who completely avoided the fallacy (averaged across the two task versions) was the lowest in the case of early adolescents $(7 \%)$. This proportion was significantly lower than in the adult group (25\%), but it did not significantly differ from the proportion of late adolescents who avoided the fallacy (19\%). Finally, there was also no significant difference between the proportion of late adolescents and adults who consistently avoided the fallacy.

Figure 2. Proportion of participants who completely avoided the fallacy in the case of traditional problems and problems with two non-representative conjuncts. Error bars represent $95 \%$ confidence interval of the means.

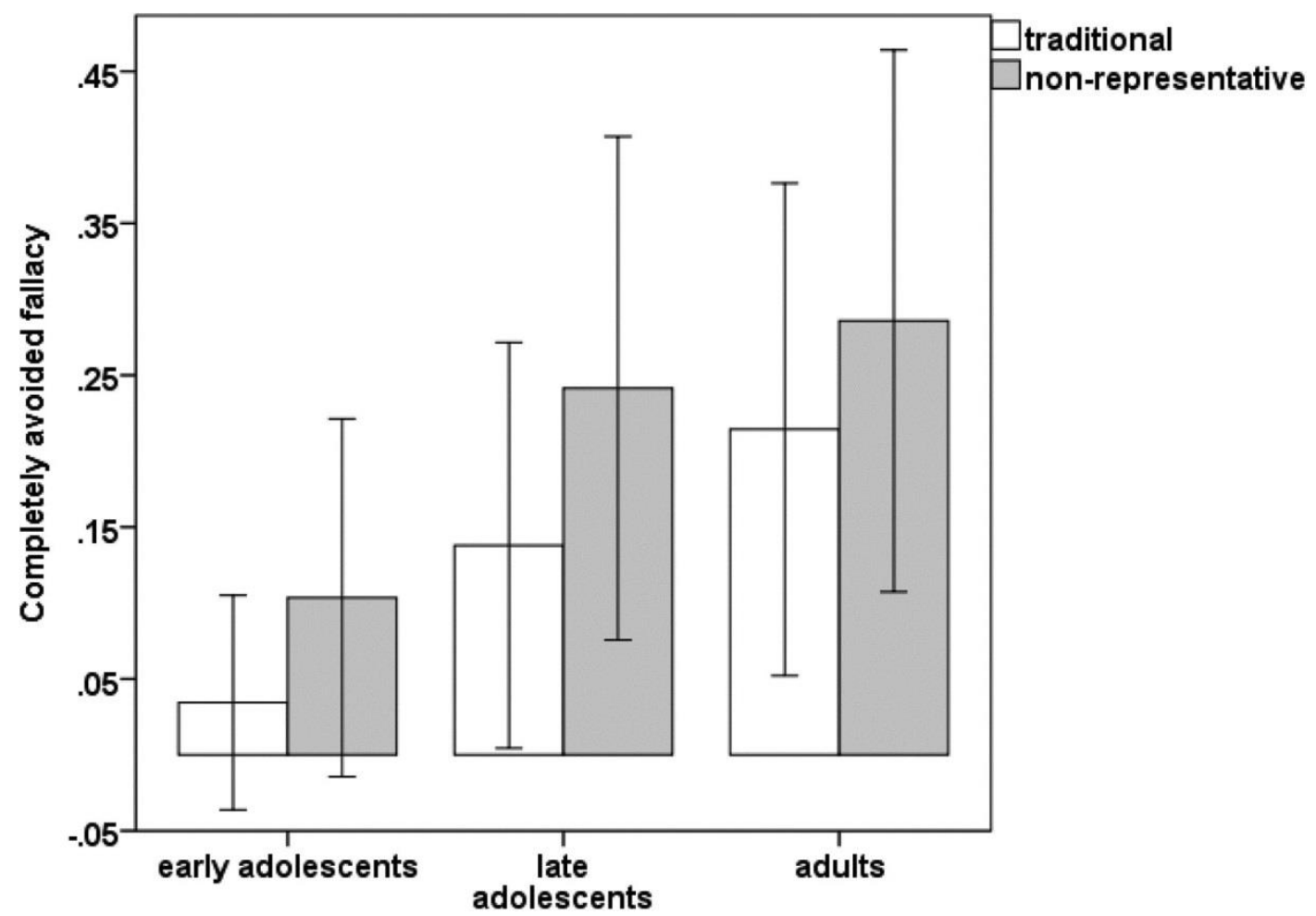


We also computed the correlation between fallacy rates across the two versions of the task. Spearman's rho correlations indicated a weak, positive relationship $\left(r_{s}(84)=.22, p=.039\right)$. That is, those participants who avoided the fallacy in the case of one version of the task (e.g., in the case of the traditional version) were somewhat more likely to avoid the fallacy in the case of the other version as well.

\section{Discussion}

In line with earlier studies (Klaczynski, 2001a; Morsanyi \& Handley, 2013), Experiment 1 indicated that the majority of adolescents (both younger and older ones) committed the conjunction fallacy, just like most of the adult participants. The manipulation of the representativeness of the conjunctive response option had a large effect on fallacy rates (i.e., fallacy rates dropped substantially). Nevertheless, fallacy rates were still relatively high in all age groups, and there was no indication that older participants benefitted from the manipulation of the representativeness of the conjunctive option to a greater extent than younger participants. Although there was a trend towards a decrease in fallacy rates with age, this did not reach significance.

When we also looked at the proportion of participants who consistently avoided the fallacy in the case of each version of the task, we found no effect of task version. However, this analysis showed a significant effect of age. This indicated that a higher proportion of adults than early adolescents managed to consistently avoid the conjunction fallacy (with late adolescents performing at an intermediate level). Nevertheless, although the manipulation was successful in making the conjunctive response option less attractive, it clearly did not result in participants' switching from a predominantly heuristic-based responding to predominantly normative reasoning. Additionally, whereas a significant minority of participants avoided the fallacy in the case of each version of the task, normative responding across the two task versions was only weakly correlated. That is, it appeared that there were very few participants, who consistently relied on the conjunction rule as the basis of their responses across both versions of the task.

In summary, Experiment 1 showed that although making the conjunctive response option less attractive reduced fallacy rates, normative performance remained weak, and the consistency of applying the conjunction rule across the two versions of the task was also relatively low. Thus, it is possible that even when the conjunction included two non-representative items, participants still remained focused on the representativeness of the response options (see, e.g., Aczel et al., 2016; Fantino et al., 1997; Fisk \& Pidgeon, 1996; Nilsson et al., 2009). Regarding age-related changes, there was some evidence of an increase in normative responding between early adolescence and adulthood. In particular, adult participants were more likely to consistently apply the conjunction rule in the case of both the traditional and the non-representative conjunct version of the task. Beside an increased understanding of the conjunction rule, it is also likely that older participants have an increased ability to recognise the relevance of this rule. Nevertheless, the results clearly show that even adult participants were reluctant to respond on the basis of the conjunction rule.

When we consider the relatively low level of normative performance, it should be noted that (similar to the original "Linda problem"), we did not state that the "item" described in the task was randomly selected from a population, and, thus, no explicit cues were provided for participants to apply the normative rules of probability (Gigerenzer, 1996; Hertwig et al., 2008; Politzer \& Noveck, 1991). We also did not instruct participants to reason logically, which is known to improve reasoning performance both in adolescence and in adulthood (e.g., Chiesi et al., 2011). Thus, it is possible that although our participants did take normative considerations into account, given that these considerations were not supported by the context of task administration, they had a limited effect on responses. 


\section{Experiment 2}

Experiment 1 showed that simply making the conjunctive response option more attractive was not enough to trigger a switch from representativeness-based to normative responding in the case of either adolescents or adults. Additionally, the effect of the manipulation was similar across age groups, although adults' reasoning was somewhat more normative in general.

In Experiment 2 we extended our investigation of developmental patterns to children. In particular, we wanted to address two questions. The first one was why a significant minority of children avoid the conjunction fallacy, although it is unlikely that they have a good understanding of the conjunction rule. The second one is, conversely, why adolescents and adults fail to use their knowledge about the conjunction rule (which many of them possess) to avoid the conjunction fallacy. We propose a single explanation for these phenomena: a developmental increase in the tendency to contextualise presented information. Given that in Experiment 1 adolescents exhibited similar response patterns to adults (although they were somewhat more susceptible to the fallacy), in the present study we just focused on the two extreme age groups: children and adults.

As we described above, Morsanyi and Handley (2008) proposed that conjunction fallacy rates increased with age during childhood, because older children were more sensitive to contextual information (i.e., relevant background knowledge, the presence or absence of other response options, etc.) than younger children. Indeed, this interpretation was also supported by another study on the conjunction fallacy with adolescents with autism (Morsanyi et al., 2010). Moreover, Morsanyi and Handley (2008) demonstrated this tendency in a number of different problems. One of these was the sunk cost fallacy (i.e., the tendency to continue to invest resources in order to achieve an aim which turned out to be worthless, because previous investment had already been made). Young children were less affected by past investments in these cases, and, thus, their decisions, which were based on the evaluation of the aim (which was not worth achieving), were more rational than that of older children.

A similar age-related change has been reported in the case of the "lost money/lost ticket" scenarios (Tversky \& Kahneman, 1981). In the "lost ticket" scenario a person loses a ticket which is worth $\$ 10$ on the way to the theatre, and they have to decide whether they would purchase another ticket for the play. In the other scenario, the person loses a $\$ 10$ note, and they have to decide whether they would still purchase a ticket which costs $\$ 10$. The typical finding with adults is that although the investment is exactly the same in both scenarios, people tend to indicate that they would be more likely to buy a ticket if they lost the money, as opposed to if they lost the ticket which they bought earlier. Webley and Plaisier (1998) found that older children (between the ages of 8 and 12) increasingly showed this bias, but young children (ages 5-6) did not.

There is further evidence to show that children are not as much influenced by the framing of objectively identical information as adults. For example, when young children are given four toys from which two toys are then subtracted (which is treated as a loss by older children and adults), they perceive this situation as identical to another scenario where they are given two toys (which is treated as a gain by older participants-see Reyna \& Ellis, 1994). Finally, children's memories of presented information are also more literal. For example, when presented with a list of semantically related words, such as bed, rest, awake, tired, dream, wake, snooze, blanket, they are less likely than adults to recall a related but non-presented word (e.g., sleep)-see, e.g., Brainerd, Reyna, and Zember (2011).

In contrast with children, adults show an excessive sensitivity to context (which has also been referred to as the "fundamental computational bias"-cf. Stanovich, 2003). Thus, we propose that a reduced susceptibility to this bias in young children could provide an explanation for the puzzling developmental findings where children's decisions appear to be more rational than that of adults. Potential explanations for susceptibility to the conjunction fallacy in adults include relying solely on the representativeness of response options (e.g., Tversky \& Kahneman, 1982), 
high sensitivity to pragmatic cues (e.g., Politzer \& Noveck, 1991), and using subjective instead of objective probability as the basis of responding (e.g., Gigerenzer, 1996). In fact, all of these explanations belong to the same broad category of a tendency to contextualise, and we expect that all of these tendencies would be less prominent in young children than in older children and adults.

Nevertheless, as we described earlier, some researchers (De Neys \& Vanderputte, 2011; Stanovich et al., 2008, 2011) have proposed that an apparent increase in conjunction fallacy rates during childhood could have arisen because existing studies used descriptions of social stereotypes which young children were unfamiliar with. In order to control for this potential confound, similar to Experiment 1, we included the representative item on its own as a choice option, and we excluded those participants from our analyses who did not mark this option as the most likely.

The most important aim of the present study, however, was to demonstrate the relative contextsensitivity of adults as compared to children. In Experiment 1, we demonstrated how adolescents and adults tend to ignore their knowledge about the conjunction rule in favour of the more salient cue of representativeness. Indeed, Kahneman and Tversky (1973) demonstrated a more extreme case of this insensitivity to probability information in their famous "engineers and lawyers" problem, and this finding has been replicated several times (e.g., De Neys \& Glumicic, 2008). In this task, participants are provided with a description of a person (a stereotypical description of an engineer) who was randomly selected from a large group of people, consisting of an extremely high proportion of lawyers and a very low proportion of engineers. In this case, the vast majority of participants judge the person to be more likely to be an engineer than a lawyer, in spite of its low probability based on frequency information. Pennycook and Thompson (2012; see also Pennycook, Trippas, Thompson, \& Handley, 2014) showed that base rate information was processed effortlessly by adults, and they also demonstrated that although educated adults readily detected the incompatibility between stereotype information and base rates, they often judged stereotype information to be more diagnostic, and, as a result, "neglected" base rates. Based on these findings, a possible interpretation of the results of Experiment 1 is that our participants considered the relevance/diagnosticity of representativeness and the conjunction rule on a case by case basis, and they generally considered representativeness to be the more relevant cue. Nevertheless, as we did not independently measure participants' knowledge of the conjunction rule in our study, it is still possible that some of our participants were unaware of this rule.

In Experiment 2, we implemented a manipulation whereby we provided explicit frequency information about the number of people belonging to the categories corresponding to $A, A \& B$, and $B$. Note that by providing frequency information, we eliminated the need for participants to apply the conjunction rule, and our task is basically a measure of whether participants prefer to base their responses on representativeness (which has to be inferred from the description), or on explicitly presented frequency information. We hypothesised that children would be more likely to rely on this explicitly presented information than adults (see, e.g., Jacobs \& Potenza, 1991), given their more literal interpretation of task information. At the same time we expected that, similarly to the typical findings on the engineers and lawyers problem, the majority of adults would ignore frequency information.

Finally, to further demonstrate the context-sensitivity of adult cognition (and how it can also be supported by context), we implemented another manipulation, which affected the context of task administration..$^{3}$ Whereas adult participants in the control condition were administered the conjunction fallacy task in the context of a study setting where they solved a series of reasoning tasks (some of which included numerical information, but they were unrelated to the conjunction fallacy), participants in the experimental condition were administered the task after a session which was designed to elicit a "mathematical mindset", and took about $30 \mathrm{~min}$. As part of this session, participants had to reason about various probabilistic events, such as repeated tosses of a coin, drawing marbles from a population with known probabilities with or without replacement, and simple, conjunct and disjunctive events derivable from a $2 \times 2$ contingency 
table. Additionally, they were explained about some fundamental rules of randomness and probability (but this did not include the conjunction rule). We hypothesised that these activities would reduce fallacy rates by making numerical information more salient and relevant.

\section{Method}

\section{Participants}

The participants were primary school children $(n=302 ; 233$ males; mean age $=9$ years 6 months), undergraduate students with a special training in probability $(n=97 ; 11$ males; mean age $=21$ years $)$, and undergraduates without a special training in probability $(n=158 ; 32$ males; mean age $=20$ years 3 months). In the pilot study, a separate sample of 145 children (83 males; mean age 9 years 7 months) and 62 undergraduate students ( 8 males; mean age $=20$ years 4 months) participated.

\section{Materials and procedure}

We created two different versions of a single task (see Table 2), and each participant was given only one of these (thus, participants' performance was scored as $1=$ committed the fallacy; $0=$ did not commit the fallacy). The tasks were based on a child-friendly version of the "Linda problem" (similar to one of the problems used by Morsanyi \& Handley, 2008). Both versions included information that the child was randomly selected from a class, in order to ensure that the normative rules of probability were relevant and appropriate to apply in the context of the task. Additionally, in the first task, the actual number of children belonging to each group which corresponded to the response options was given. In the case of both tasks, the response options included the representative item alone, a representative-non-representative conjunction, and the non-representative item alone. Similar to Experiment 1, the measure of fallacy ${ }^{4}$ was whether participants rated the conjunction as more probable than the non-representative item alone. Primary school children and university students without a training were administered the task as part of a bigger testing session. University students with a training were administered the task after a training session, together with some other problems.

Table 2. Different versions of the problem used in Experiment 2.

\begin{tabular}{||l||l||}
\hline With numerical information & $\begin{array}{l}\text { Without numerical } \\
\text { information }\end{array}$ \\
\hline \hline $\begin{array}{l}\text { From a class of } 30 \text { children, 12 play football, } 4 \text { play music and } 2 \\
\text { play both football and music. }\end{array}$ & $\begin{array}{l}\text { From a class of } 30 \\
\text { children ... }\end{array}$ \\
\hline \hline
\end{tabular}

the teacher picked at random a child for an assignment. Tom was chosen. Tom lives in a house with a large garden. His favourite school subject is playtime. He has many friends and he loves sport. He collects football stickers. Mark the following statements with numbers 1 to 3 according to how likely they are to be true. Mark the statement which is the most likely to be true with 1 , and the one which is the least likely to be true with 3.
a. Tom plays football.
b. Tom plays football and music.
c. Tom plays music. 
Before the main study, a pilot study was conducted (this was also embedded in a bigger testing session including other tasks). In this study, the same problem was used as in the condition with numerical information. However, in this version, the representative item alone (i.e., "Tom plays football") was not included, but it was replaced by the statement "Tom has a younger sibling". The aim of this preliminary study was to confirm that the content of the task was appropriate for children.

\section{Results}

We report the results of the pilot study first. As expected, both children and adults were highly susceptible to the conjunction fallacy. Indeed, $82 \%$ of the children $(S D=.38)$, and $89 \%$ of the adult participants $(S D=.32)$ committed the fallacy. A Mann-Whitney $U$ test indicated that fallacy rates did not differ between the two groups $(p=.233) . \underline{5}$

Now we turn to the results of the main study. First, in order to check whether children were as able to recognise the stereotype cued by the description as adults, we compared the proportion of participants who identified the representative option as the most likely across the two age groups (primary school/university), using a chi-square test. In the primary school sample, $86.4 \%$ of the children identified the representative option to be the most likely, as opposed to $94.5 \%$ of participants in the university student sample. The difference between age groups was significant $\left(x^{2}(d f=1, N=554)=10.06, p=.002\right)$. We also checked whether the proportion of children who marked the representative option as the most likely changed with age using Pearson productmoment correlation. The relationship was significant, indicating a moderate increase with age in the child sample $(r(300)=.22, p<.001)$. Finally, given that in the case of the task with numerical information, it was possible that children selected the representative option as the most likely purely on the basis of the numerical information provided, we have additionally compared the proportion of children who selected the representative option as the most probable across the two versions of the task. A chi-square test indicated no significant difference $(p=.61)$ between the proportion of children who marked this option as the most probable in the case of the task where numerical information was provided $(86 \%)$ versus in the task where this information was not provided (87\%).

The purpose of our next analysis was to compare fallacy rates across groups when numerical information was present/absent (see Figure 3). Similar to Experiment 1, in this analysis we only included those participants who marked the representative option as the most likely. Thus, we excluded 41 primary school students, 9 university students from the control condition, and 5 university students from the mathematical mindset condition.

Figure 3. The effect of the presence/absence of numerical information on fallacy rates across groups. Error bars represent $95 \%$ confidence interval of the means.

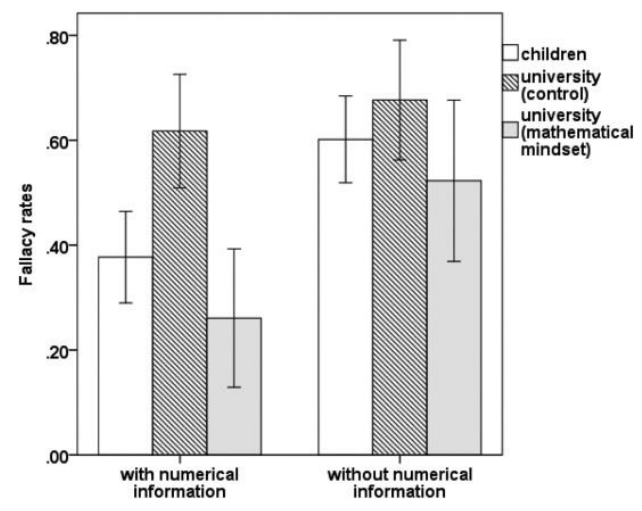


Given the categorical nature of the data, a logistic regression analysis was conducted with fallacy committed/not committed as a dependent variable. The predictor variables were numerical information (present/absent) and level of education (primary school students/university students-control /university students-mathematical mindset). The three levels of the latter variable were transformed into dummy variables, with university students (mathematical mindset) considered as a reference. The logistic regression model was statistically significant $\left(x^{2}(3\right.$, $N=493$ ) $=32.01, p<.001$ ). About $62 \%$ of cases were correctly classified (fallacy committed: $55 \%$; fallacy not committed: $69 \%$ ) by the full regression model. The relative influence of the predictors is shown in Table 3 . Fallacy rates significantly increased when numerical information was absent (i.e., without numerical information, participants were twice as likely to commit the fallacy). ${ }^{6}$ There was no significant difference between university students (mathematical mindset) and primary school students. Fallacy rates were lower in university students (mathematical mindset) and primary school students than university students in the control condition. Specifically, students in the control condition were three times more likely to give fallacious responses than students with a "mathematical mindset" and primary school students. Finally, the interaction between numerical information and mathematical mindset was also significant $\left(X^{2}(5\right.$, $N=493)=36.50, p<.001)$. Indeed, students with both a mathematical mindset and numerical information were the least likely to commit the fallacy. Specifically, for the task without numerical information, fallacy rates were $60 \%, 68 \%$, and $52 \%$, for the primary school students, university students (control), and university students (mathematical mindset), respectively. In the case of the task with numerical information, fallacy rates were $38 \%, 62 \%$, and $26 \%$, respectively.

Table 3. Logistic regression with conjunction fallacy as dependent variable (no fallacy/fallacy).

\begin{tabular}{||l||c||c||c||c||c||}
\hline Predictors & $B$ & Wald & $p$ & $\begin{array}{c}\text { Odds } \\
\text { ratio }\end{array}$ & $95 \% \mathrm{Cl}$ \\
\hline \hline Numerical information & 0.74 & 15.47 & $<.001$ & 2.09 & $\begin{array}{c}1.45- \\
3.02\end{array}$ \\
\hline \hline Educational level & & 17.32 & $<.001$ & & \\
\hline \hline Primary school & 0.39 & 2.31 & .129 & 1.47 & $\begin{array}{c}0.89- \\
2.42\end{array}$ \\
\hline \hline University students (control) & 1.09 & 15.06 & $<.001$ & 2.98 & $\begin{array}{c}1.72- \\
5.17\end{array}$ \\
\hline \hline $\begin{array}{l}\text { Mathematical mindset } \times \text { numerical } \\
\text { information }\end{array}$ & 1.32 & 6.29 & $<.001$ & 3.10 & $\begin{array}{c}1.28- \\
7.52\end{array}$ \\
\hline \hline
\end{tabular}

Note: Overall model evaluation: Likelihood ratio test: $X^{2}=32.09, d f=3, p<.001$. Goodness-of-fit test: Hosmer \& Lemeshow: $\chi^{2}=2.72, \mathrm{df}=4, p=.61$; Nagelkerke $R^{2}=.08$. Correct classification: $62 \%$.

\section{Discussion}

The purpose of this study was to understand why children perform relatively well on conjunction fallacy problems (despite their relatively poor knowledge of the rules of probability), and why 
adults perform relatively poorly (despite their generally better understanding of probabilities). We suggested that both of these puzzling phenomena could be explained by differences between children and adults in their tendency to use contextual information, as opposed to a tendency to rely on a literal interpretation of explicitly presented information.

Regarding children, some researchers (e.g., Stanovich et al., 2008, 2011) suggested that their seemingly good performance could be just a side-effect of using unfamiliar stereotypes in the problem descriptions. Indeed, our results provided some evidence for this claim. Specifically, a lower proportion of children than adults marked the representative item as the most likely option, and there was also a weak, but reliable correlation between children's age and their tendency to mark this option as the most likely. Nevertheless, the vast majority $(86.4 \%)$ of children in our sample marked this option as the most likely, and this was the case even when frequency information was not provided. Moreover, although we only included those children in our analyses who marked the representative option as the most likely, their overall performance was still relatively good (indeed, significantly better than that of university students' in the control condition). It is also important to note that we replicated an earlier finding (Morsanyi \& Handley, 2013) which showed that children performed at the same level as adults on the traditional version of the task. Indeed, in the present experiment, this was still the case when we compared children with university students who participated in a session which was designed to elicit a "mathematical mindset".

Another remarkable finding regarding children's performance is that they showed high sensitivity to explicitly presented numerical information, similar to adults in the "mathematical mindset" condition (see also Jacobs \& Potenza, 1991). Arguably, in general, adults are better able to use probability information than children (e.g., Chiesi et al., 2011), and we do not want to challenge this assumption. For example, Jacobs and Potenza (1991) showed that although children were more sensitive to base rates than adults in the case of problems with a conflict between representativeness and base rates, their justification of their responses did not indicate a conscious reliance on normative considerations.

We propose that the reason for children's relatively good performance is that (as compared to adults) they are characterised by an increased sensitivity to explicitly presented information (including irrelevant surface characteristics), and reduced sensitivity to information which can be inferred from presented information (specifically, a social stereotype, in this case). This interpretation is consistent with results from adolescents with autism in the case of the conjunction fallacy task (Morsanyi et al., 2010), and more general findings regarding children's reasoning development (e.g., Brainerd et al., 2011; Reyna \& Ellis, 1994; Webley \& Plaisier, 1998). These findings are also in line with the general claim of the fuzzy-trace theory (e.g., Reyna \& Brainerd, 2011) that adults tend to prefer "gist-based processing" (i.e., processing for meaning rather than verbatim detail), whereas children are more literal in their interpretation, especially if they do not have to recall details from memory. What is particularly remarkable, is that children disregarded stereotype information, although they marked the representative option as the most likely. Thus, they activated the stereotype, and used it as the basis of finding the most likely response. Nevertheless, this knowledge was not utilised as much when they evaluated the conjunctive option.

A few potential issues regarding the contextualisation account should be noted. Although our child participants demonstrated familiarity with the relevant stereotypes, this does not necessarily mean that the degree of familiarity was the same across children and adults. ${ }^{\underline{ }}$ It is quite plausible to suppose that children do not consistently use stereotype information, because the retrieval process is effortful and/or because keeping this activated information in mind requires effort. However, even if it was only the stereotype identification stage that was difficult for children, this would still be in line with our conclusion that the contextualisation process is more effortful for children than for adults. Note that this is in contrast with some mainstream accounts of the development of reasoning skills. Consider for example this commentary on Jacobs and Potenza (1991) by Toplak et al. (2014, p. 1038): 
... younger children are prevented from showing the bias because they do not know the stereotype. Older children, however, will be familiar with this stereotype. This will artificially create a pattern looking like a non-normative bias increasing with age. In fact, it is not really a processing bias increasing with age, but the knowledge that makes showing the bias possible that increases with age.

Based on our results, we can conclude that, in fact, relevant knowledge is available, and it is indeed the processes involved in retrieving and using this knowledge that differ between children and adults (i.e., these processes are less fluent/more effortful in the case of children). This is also consistent with the existence of a positive correlation between certain reasoning biases and cognitive capacity in young children (Morsanyi \& Handley, 2008) and in adolescents with autism (Morsanyi et al., 2011).

Regarding the results with adults, probably the most striking finding is that students in the control condition did not use explicitly presented frequency information. More precisely, although the presentation of frequency information slightly reduced fallacy rates, the majority of these participants still committed the fallacy. This result is especially interesting, given that it was explicitly stated that the person who was described in the task was randomly selected from a group, thus, the argument that the rules of objective probability are irrelevant (e.g., Gigerenzer, 1996) does not apply in the case of these tasks. Although it seems curious that explicitly presented frequency information might be ignored, as we described earlier, similar findings have been reported in the case of the famous "engineers and lawyers" problem of Kahneman and Tversky (1973). The fact that participants did not utilise frequency information is also entirely consistent with the results of Experiment 1, where participants' normative performance did not improve when the conjunctive response option was made much less attractive (at least participants did not consistently rely on the conjunction rule even in this case).

The current results are also consistent with Pennycook and Thompson (2012; see also Pennycook et al., 2014) who found that, although reasoning with base rates was routine and relatively effortless in the case of adults, and if there was a conflict between base rates and stereotype information, people readily detected this, whether they decided to rely on base rates or stereotype information depended on the context of task administration. Similarly, in the current study, for adults who participated in a session emphasising the importance of probability information, the relevance of numerical information was more evident, and their responses changed accordingly. However, although it is very likely that adults in the control condition also noted the frequency information provided in the task, they did not rely on this, as they judged it to be less relevant than the representativeness of response options. The assumption that reasoning with base rates requires little effort (cf. Pennycook \& Thompson, 2012) is also supported by the fact that children readily used this information. In fact, when this information conflicted with representativeness, children preferentially based their responses on base rates.

It could be argued that a weakness of our design is that, although the single representative item was twice as likely as the conjunctive option (on the basis of base rates), maybe this difference was not large enough to provide a convincing cue for participants to switch from a representativeness-based response to a base rate-based response. It is possible that adult participants would have showed a greater sensitivity to base rates if the differences between frequencies linked to each response option were larger. Future studies could compare sensitivity to base rates between children and adults while manipulating frequency information (i.e., how extreme base rates are). At the same time, it is remarkable that inducing a "mathematical mindset" had a dramatic effect on the performance of adult participants (i.e., leading to a threefold reduction in fallacy rates). When frequency information was made relevant and, thus, salient by presenting it in the context of a probability training, adults shifted their preference from a general tendency to judge the response options on the basis of representativeness to a tendency to judge them on the basis of probability. This demonstrates the two faces of context-sensitivity: leading to both an ability to utilise subtle cues, and to a susceptibility to rely on irrelevant information. 
To summarise our interpretation regarding the differences between children and adults; first, it is important to note that without the frequency information primary school children were just as susceptible to the fallacy as adults (as also evidenced by the results of our pilot study, and as previously reported by Morsanyi \& Handley, 2013). Thus, based on the traditional conjunction fallacy task alone, there is no evidence that stereotype information would affect children's judgments less strongly. Nevertheless, we propose that the underlying processes are different. Some adults avoid the fallacy because they spontaneously recognise the relevance of the conjunction rule. By contrast, children who avoid the fallacy find the conjunctive option improbable for other reasons. For example, they might simply think that "children who play football usually don't play music", so this is an unlikely event. It is not that they are completely unaffected by the stereotype cued by the description (as they all marked the representative single option as the most likely), but this is less salient or available than other cues (such as the actual content of the response options and frequency information) or they might find it more difficult to link the description of the child to the conjunctive response option. When the task is presented together with frequency information, although adults obviously have no problem with processing frequencies (cf. Pennycook \& Thompson, 2012), they probably find this information irrelevant, and base their responses preferably on stereotype information, unless the relevance of base rates is highlighted (for example, by inducing a "mathematical mindset"). Many children, however, will take frequency information at face value, and this is why their likelihood ratings for the conjunctive options will be lower.

\section{General discussion}

In two experiments, we tested the "contextualisation" account (Morsanyi \& Handley, 2008) of developmental changes in the conjunction fallacy. Although there is a vast literature dealing with the conjunction fallacy phenomenon, so far there are only a handful of studies that have investigated developmental changes in responding. These studies have established that on versions of the tasks which have a similar format to the famous "Linda problem" (Tversky \& Kahneman, 1982), fallacy rates tend to increase during childhood (Davidson, 1995; Morsanyi \& Handley, 2008), and young children show similar performance to adults (Morsanyi \& Handley, 2013). These findings are surprising, given existing evidence that young children are less sensitive to probability information in pictorial conjunction fallacy problems than older children and adults (Chiesi et al., 2008), and when the linguistic ambiguity inherent in the task is eliminated in pictorial problems, children are less able to apply the conjunction rule than adults (Fisk \& Slattery, 2005).

In Experiment 1, we showed that in adolescence and adulthood, fallacy rates dropped considerably when the conjunctive option was non-representative. In this case, approximately half of our participants did not commit the fallacy. Nevertheless, making the conjunctive option less attractive did not increase the number of participants who consistently relied on the conjunction rule. In other words, although the manipulation had a large effect on response patterns, it did not prompt a switch from representativeness-based to normative responding. Thus, it is likely that although the conjunctive option was rated as less probable when it contained two non-representative options as compared to when one of the conjuncts was representative, our participants mainly relied on the representativeness of the response options in the case of both types of task. Indeed, consistent normative responding in the case of both versions of the task was rare in both adolescents and adults, although adults performed better than adolescents.

Experiment 2 provided further evidence that adults' reasoning was primarily determined by contextual information. When the need to apply the conjunction rule was eliminated by explicitly providing frequency information, the majority of university students without training still disregarded probabilities. This clearly demonstrates that most of these participants perceived frequency information to be irrelevant to their judgments. However, when a "mathematical mindset" was induced in participants, and, thus, numerical information appeared relevant, participants' performance improved, especially when frequency information was explicitly 
provided. Although our manipulations that were aimed at cueing analytic processing in Experiments 1 and 2 remained remarkably inefficient (with the exception of a combination of frequency information and a general training in probabilistic reasoning), it is possible that if we used more sensitive measures (e.g., inspection times, or ratings of response confidence), we could have observed some effect of these manipulations. Indeed, reasoners sometimes show evidence that they experience a conflict between heuristic and analytic considerations, even when they give heuristic-based responses (e.g., De Neys, 2012). That is, although their responses showed a dominant influence of representativeness, it is possible that these responses were generated less fluently and confidently by our participants, as a result of our manipulations.

In terms of developmental investigations, previous studies with children (De Neys \& Feremans, 2013) and adolescents (e.g., De Neys, Cromheeke, \& Osman, 2011) showed that younger reasoners tend to be worse at detecting conflict than adults. Based on our results, it is possible that, at least in the case of children, weaker evidence for conflict could be to some extent attributed to relatively weaker influence of heuristic cues (although normative considerations are also likely to have a smaller effect in the case of children). Future studies could also investigate whether various manipulations (e.g., manipulating the salience of relevant stereotypes or frequency information) could affect conflict detection ability in children and adults differentially. For example, it is possible that in the case of the task with explicit frequency information in Experiment 2, children would experience a higher level of conflict if the relevant stereotypes were made more salient. This would be especially interesting if, at the same time, the manipulation would increase heuristic responding. By contrast, the same manipulation might result in lower levels of experienced conflict in adults.

Regarding children's performance, our findings lend some support to claims that they might be less able to recognise relevant stereotypes cued by the tasks than adults (cf. De Neys \& Vanderputte, 2011; Stanovich et al., 2008, 2011), or that they might be less able to use these stereotypes as the basis of their judgments. Nevertheless, even when this confound was eliminated, children showed a more literal, and, thus, more normative interpretation of the response options than adults. Most strikingly, when frequency information was presented, children switched from a tendency to commit the fallacy to a tendency to avoid the fallacy, which did not happen in the case of adults, unless the relevance of probability information was specifically highlighted to them.

Osman and Stavy (2006) discussed the role of bottom-up and top-down saliency in people's interpretation of tasks, where bottom-up saliency is based on the objective characteristics of presented stimuli, and top-down saliency is based on participants' knowledge of (and previous experiences with) the stimuli. Our findings suggest that there is a developmental shift from childhood to adulthood from a dominant reliance on bottom-up, to a dominant reliance on topdown saliency. Specifically, in our view, whereas during childhood, the most important developmental achievement seems to be an increasing ability to integrate contextual information (e.g., Morsanyi \& Handley, 2008), by adolescence contextualisation becomes fairly automatic and effortless, and the main driving force of developmental change is an increasing knowledge of normative rules, and an increasing ability to retrieve and implement these rules when needed (e.g., Chiesi et al., 2011; Stanovich et al., 2008). Together these changes can explain the inverted U-shaped developmental trajectory of judgment and reasoning fallacies between childhood and adulthood, which were observed by Morsanyi and Handley (2013). These changes could be related to the development of executive functioning skills (i.e., the ability to ignore salient, but irrelevant distracters when processing higher level information-e.g., Richland, Morrison, \& Holyoak, 2006), and to the accumulation of knowledge which becomes more ingrained and, thus, easier to activate with development. At the same time, there is a shift from literal to pragmatic (Chiesi et al., 2008; Feeney et al., 2004), non-contextualised to contextualised (Evans, 2011; Morsanyi \& Handley, 2008) and verbatim to gist processing (e.g., Reyna \& Brainerd, 2011). 
Although we provided a general framework for understanding developmental changes in the conjunction fallacy, as a limitation of this paper, we should highlight some issues that were not addressed by the current experiments. It has been long recognised (see Reyna, 1991) that to some extent conjunction fallacy problems correspond to Piaget's (1965) class inclusion problems. Nevertheless, the issue of whether some young children commit the fallacy because they misunderstand class relationships has never been addressed. More precisely, Fisk and Slattery (2005) investigated children's class inclusion performance together with their performance on a version of the conjunction fallacy task, and found that these were unrelated. However, in their version of the conjunction problem, the potential class inclusion confound was already eliminated.

We also did not address the role of pragmatic understanding in performance differences between children and adults (see, e.g., Chiesi et al., 2008; Feeney et al., 2004; Noveck, 2001). For example, it could be expected that including the "A\&notB" option together with the "A" option would have a greater effect on the performance of adults than that of children. Nevertheless, in our view, the increasing sensitivity to pragmatic cues is just one aspect of a more general developmental trend to be more influenced by context as children get older.

In Experiment 2, we demonstrated how adults' interpretation of the same task information changed depending on the context of task administration (i.e., whether frequency information was made salient). Indeed, as we discussed earlier, there is ample evidence for the sensitivity of adult participants to contextual information. One procedure that has been used in several studies to demonstrate this flexibility in handling information is to instruct participants to reason on the basis of logic vs. to reason on the basis of their intuitions or feelings (e.g., Denes-Raj \& Epstein, 1994; Klaczynski, 2001b; Morsanyi, Primi, Chiesi, \& Handley, 2009). In these cases, participants in the two instruction conditions show different response patterns. In line with our claim that context-sensitivity increases with development, Chiesi et al. (2011) found that instructions had a greater effect on adults' as compared to children's responses, and this developmental change appeared to be related to increases in cognitive capacity, and the knowledge of normative rules. Given that instructions can be used to highlight certain task characteristics, we would expect that detailed instructions to treat the conjunction problems as tests of probabilistic reasoning would reduce fallacy rates, and this improvement would be greater in the case of adults than in the case of children. Nevertheless, all the above suggestions remain to be tested in future studies.

Our findings regarding reduced context-sensitivity in children relative to adolescents and adults are in line with some earlier investigations, where other paradigms were used, such as the framing effect (Reyna \& Ellis, 1994), the sunk cost fallacy (Morsanyi \& Handley, 2008), and the lost money/lost ticket scenarios (Webley \& Plaisier, 1998), and problems with a conflict between base rates and stereotype information (Jacobs \& Potenza, 1991). However, it is important that future studies extend these systematic investigations of judgment and reasoning skills in young children to other tasks, and confirm the generalisability of our developmental claims. Our findings can also be linked to studies that reported increases in intuitive errors with experience (Reyna, Chick, Corbin, \& Hsia, 2014) and education (e.g., Morsanyi, Handley, \& Serpell, 2013; Morsanyi et al., 2009; Van Dooren, De Bock, Hessels, Janssens, \& Verschaffel, 2005).

A final aspect of our results that is worth noting is their relevance to the debate on human rationality (e.g., Elqayam \& Evans, 2011; Stanovich \& West, 2000), and to the question of whether people become more rational (and better reasoners) with development. The implications of our findings are very simple and clear. The potential for normative, rule-based reasoning increases with development. Nevertheless, whereas context-sensitivity is a key characteristic of human cognition, and it is a major contributor to the flexibility of adult thinking, in some cases it can lead to response patterns that violate some very basic normative rules. When this happens, young children's responses appear to be at least as normative and rational (if not more so) as that of adults'. Thus, the "illusion of replacement" (cf. Reyna \& Ellis, 1994) is indeed an illusion. Adolescents and adults will rely on normative reasoning only when this seems to be the most appropriate as indicated by contextual cues. 


\section{Funding}

This research, and the writing of this paper, was partially supported by a UK Economic and Social Research Council post-doctoral fellowship [PTA-026-27-2989] to KM.

1. Beside the conjunction fallacy, participants with autism have also been found to show reduced susceptibility to framing effects (De Martino, Harrison, Knafo, Bird, \& Dolan, 2008)

2. These tasks consisted of reasoning problems that required the use of numerical information that were piloted for the purposes of another study.

3. Throughout the paper, we use the term "context" in a very broad sense, but in general we refer to additional information presented together with key task information. Thus, within a conjunction fallacy task, context refers to the description of the person in the task, the response options included, as well as the overall context of task administration (e.g., following a training session in probabilistic reasoning).

4. As we explained earlier, the task with numerical information is not a conjunction fallacy task, as knowledge of the conjunction rule is not necessary for participants to give a normative response. Nevertheless, based on frequency information, rating the conjunctive response as more likely than the single non-representative response was non-normative. Thus, for the sake of simplicity, we referred to this type of response as a "fallacious" response.

5. Given that the gender distribution of the child and adult samples was different, we also compared fallacy rates across male and female participants. Gender was not a significant predictor of conjunction fallacy rates $(p=.310)$. As gender differences are not customarily reported in this literature, we do not report these analyses in the main text.

6. Given that the gender distribution of the child and adult samples was markedly different, we also run this analysis with the inclusion of gender. Gender was not a significant predictor of conjunction fallacy rates $(p=.494)$, and the inclusion of gender did not substantially change the other results.

7. We thank Wim De Neys for raising this issue.

\section{References}

Aczel, B., Szollosi, A., \& Bago, B. (2016). Lax monitoring versus logical intuition: The determinants of confidence in conjunction fallacy. Thinking \& Reasoning, 22, 99-117.

Adler, J. (1984). Abstraction is uncooperative. Journal for the Theory of Social Behaviour, 14, 165-181.

Ahn, W., \& Bailenson, J. (1996). Causal attribution as a search for underlying mechanisms: An explanation of the conjunction fallacy and the discounting principle. Cognitive Psychology, 31, 82-123.

Brainerd, C.J., Reyna, V.F., \& Zember, E. (2011). Theoretical and forensic implications of developmental studies of the DRM illusion. Memory \& Cognition, 39, 365-380.

Chiesi, F., Gronchi, G., \&, Primi, C. (2008). Age-trend related differences in task involving conjunctive probabilistic reasoning. Canadian Journal of Experimental Psychology, 62, 188-191.

Chiesi, F., Primi, C. \& Morsanyi, K. (2011). Developmental changes in probabilistic reasoning: The role of cognitive capacity, instructions, thinking styles and relevant knowledge. Thinking \& Reasoning, 17, 315-350.

Davidson, D. (1995). The representativeness heuristics and the conjunction fallacy in children's decision making. Merrill Palmer Quarterly, 41, 328-346.

De Martino, B., Harrison, N. A., Knafo, S., Bird, G., \& Dolan, R. J. (2008). Explaining enhanced logical consistency during decision making in autism. The Journal of Neuroscience, 28, 10746-10750.

Denes-Raj, V., \& Epstein, S. (1994). Conflict between intuitive and rational processing: When people behave against their better judgment. Journal of Personality and Social Psychology, 66, 819-829. 
De Neys, W. (2012). Bias and conflict a case for logical intuitions. Perspectives on Psychological Science, 7, 28-38.

De Neys, W., Cromheeke, S. \& Osman, M. (2011). Biased but in doubt: Conflict and decision confidence. PLOS ONE, 6: e15954. doi:10.1371/journal.pone.0015954

De Neys, W., \& Feremans, V. (2013). Development of heuristic bias detection in elementary school. Developmental Psychology, 49, 258-269.

De Neys, W., \& Glumicic, T. (2008). Conflict monitoring in dual process theories of reasoning. Cognition, 106, 1248-1299.

De Neys, W., \& Vanderputte, K. (2011). When less is not always more: Stereotype knowledge and reasoning development. Developmental Psychology, 47, 432-441.

Elqayam, S. \& Evans, St.B.T. (2011). Subtracting 'ought' from 'is': Descriptivism versus normativism in the study of the human thinking. Behavioral and Brain Sciences, 34, 233248.

Evans, J.S.B.T. (2011). Dual-process theories of reasoning: Contemporary issues and developmental applications. Developmental Review, 31, 86-102.

Fantino, E., Kulik, J., Stolarez-Fantino, S., \& Wright, W. (1997). The conjunction fallacy: A test of averaging hypotheses. Psychonomic Bulletin \& Review, 4, 96-101.

Feeney, A., Scrafton, S., Duckworth, A. \& Handley, S.J. (2004). The story of some: Everyday pragmatic inference by children and adults. Canadian Journal of Experimental Psychology, 58, 90-101.

Fisk, J. E. \& Pidgeon, N. (1996). Component probabilities and the conjunction fallacy: Resolving signed summation and the low component model in a contingent approach. Acta Psychologica, 94, 1-20.

Fisk, J. E., \& Slattery, R. (2005). Reasoning about conjunctive probabilistic concepts in childhood. Canadian Journal of Experimental Psychology, 59, 168-178.

Gigerenzer, G. (1996). On narrow norms and vague heuristics: A rebuttal to Kahneman and Tversky. Psychological Review, 103, 592-596.

Gilovich, T., Griffin D., \& Kahneman, D. (Eds.). (2002). Heuristics and biases: The psychology of intuitive judgment. Cambridge, UK: Cambridge University Press.

Happé , F. \& Frith, U. (2006). The weak coherence account: detail-focused cognitive style in autism spectrum disorders. Journal of Autism and Developmental Disorders, 35, 5-25.

Hertwig, R., Benz, B., \& Krauss, S. (2008). The conjunction fallacy and the many meanings of and. Cognition, 108, 740-753.

Jacobs, J. E., \& Potenza, M. (1991). The use of judgment heuristics to make social and object decision: A developmental perspective. Child Development, 62, 166-178.

Kahneman, D., \& Frederick, S. (2002). Representativeness revisited: Attribute substitution in intuitive judgement. In T. Gilovich, D. Griffin, \& D. Kahneman (Eds.), Heuristics and biases: The psychology of intuitive judgment (pp. 49-81). New York: Cambridge University Press.

Kahneman, D., \& Tversky, A. (1973). On the psychology of prediction. Psychological Review, 80, 237-251.

Klaczynski, P. A. (2001a). The influence of analytic and heuristic processing on adolescent reasoning and decision making. Child Development, 72, 844-861.

Klaczynski, P. A. (2001b). Framing effects on adolescent task representations, analytic and heuristic processing, and decision making: Implications for the normativedescriptive gap. Journal of Applied Developmental Psychology, 22, 289-309.

Morsanyi, K., \& Handley, S.J. (2008). How smart do you need to be to get it wrong? The role of cognitive capacity in the development of heuristic-based judgment. Journal of Experimental Child Psychology, 99, 18-36.

Morsanyi, K., \& Handley, S.J. (2013). Heuristics and biases - Insights from developmental studies. In: P. Barrouillet \& C. Gauffroy (Eds.) The development of thinking and reasoning. (pp. 122-149). Hove, UK: Psychology Press.

Morsanyi, K., Handley, S.J. \& Evans J.S.B.T. (2010). Decontextualised minds: Adolescents with autism are less susceptible to the conjunction fallacy than typically developing adolescents. Journal of Autism and Developmental Disorders, 40, 1378-1388. 
Morsanyi, K., Handley, S.J. \& Serpell, S. (2013). Making heads or tails of probability. An experiment with random generators. British Journal of Educational Psychology, 83, 379395.

Morsanyi, K., Primi, C., Chiesi, F., Handley, S.J. (2009). The effects and side-effects of statistics education. Psychology students' (mis-)conceptions of probability. Contemporary Educational Psychology, 34, 210-220.

Nilsson, H., Winman, A., Juslin, P. \& Hansson, G. (2009). Linda is not a bearded lady: Configural weighting and adding as the cause of extension errors. Journal of Experimental Psychology: General, 138, 517-534.

Noveck, I.A. (2001). When children are more logical than adults: Investigations of scalar implicature. Cognition, 78,165-188.

Osman, M. \& Stavy, R. (2006). Development of intuitive rules: evaluating the application of the dual-system framework to understanding children's intuitive reasoning. Psychonomic Bulletin \& Review, 13, 935-953.

Pennycook, G. \& Thompson, V. A. (2012). Reasoning with base-rates is routine, relatively effortless, and context dependent. Psychonomic Bulletin \& Review, 19, 528-534.

Pennycook, G., Trippas, D., Thompson, V. A., \& Handley, S. J. (2014). Base rates: Both neglected and intuitive. Journal of Experimental Psychology: Learning, Memory, and Cognition, 20, 544-554.

Piaget, J. (1965). The child's conception of number. New York: Norton.

Politzer, G., \& Noveck, I. A. (1991). Are conjunction rule violations the result of conversational rule violations? Journal of Psycholinguistic Research, 20, 83-103.

Reyna, V.F. (1991). Class inclusion, the conjunction fallacy, and other cognitive illusions. Developmental Review, 11, 317-336.

Reyna, V.F., \& Brainerd, C.J. (2011). Dual processes in decision making and developmental neuroscience: A fuzzy-trace model. Developmental Review, 31, 180-206.

Reyna, V. F., Chick, C. F., Corbin, J. C., \& Hsia, A. N. (2014). Developmental reversals in risky decision-making: Intelligence agents show larger decision biases than college students. Psychological Science. Advance online publication. doi: 10.1177/0956797613497022.

Reyna, V.F. \& Ellis, S.C. (1994). Fuzzy-trace theory and framing effects in children's risky decision making. Psychological Science, 5, 275-279.

Richland, L.E., Morrison, R.G., \& Holyoak, K.J. (2006). Children's development of analogical reasoning: Insights from scene analogy problems. Journal of Experimental Child Psychology, 94, 249-273.

Stanovich, K. E. (2003). The fundamental computational biases of human cognition: Heuristics that (sometimes) impair decision making and problem solving. In J. E. Davidson \& R. J. Sternberg (Eds.). The psychology of problem solving (pp. 291-342). New York: Cambridge University Press.

Stanovich, K. E., \& West, R. F. (2000). Individual differences in reasoning: Implications for the rationality debate? Behavioral and Brain Sciences, 23, 645-665.

Stanovich, K. E, \& West. R. F. (2008). On the relative independence of thinking biases and cognitive ability. Journal of Personality and Social Psychology, 94, 672-695.

Stanovich, K. E., Toplak, M. E., \& West, R. F. (2008). The development of rational thought: A taxonomy of heuristics and biases. Advances in child development and behaviour, 36, 251-285.

Stanovich, K. E., West, R. F., \& Toplak, M. E. (2011). The complexity of developmental predictions from dual process models. Developmental Review, 31, 103-118.

Tentori, K., Bonini, N., \& Osherson, D. (2004). The conjunction fallacy: A misunderstanding about conjunction? Cognitive Science, 28, 467-477.

Tentori, K. and Crupi, V. (2012). On the conjunction fallacy and the meaning of and, yet again: A reply to Hertwig, Benz, and Krauss (2008), Cognition, 122, 123-134.

Toplak, M. E., West, R. F., \& Stanovich, K. E. (2014). Rational thinking and cognitive sophistication: Development, cognitive abilities, and thinking dispositions. Developmental Psychology, 50, 1037-1048.

Tversky, A. \& Kahneman, D. (1981). The framing of decisions and the psychology of choice. Science, 211, 453-458. 
Tversky, A. \& Kahneman, D. (1982). Judgments of and by representativeness. In D. Kahneman, P. Slovic \& A. Tversky (Eds.), Judgment under uncertainty: Heuristics and biases. Cambridge, UK: Cambridge University Press.

Tversky, A., \& Kahneman, D. (1983). Extentional versus intuitive reasoning: The conjunction fallacy in probability judgment. Psychological Bulletin, 90, 293-315.

Van Dooren, W., De Bock, D., Hessels, A., Janssens, D., Verschaffel, L. (2005). Remedying secondary school students' illusion of linearity: developing and evaluating a powerful learning environment. In: Verschaffel L., e.a. (Eds.), Powerful environments for promoting deep conceptual and strategic learning (Studia paedagogica, 41) (pp. 115-132). Leuven: Universitaire Press.

Webley, P., \& Plaisier, Z. (1998). Mental accounting in childhood. Children's Social and Economics Education, 3, 55-64.

Wedell, D. H., \& Moro, R. (2008). Testing boundary conditions for the conjunction fallacy: Effects of response mode, conceptual focus and problem type. Cognition, 107, 105-136.

Wells, G. L. (1985). The conjunction error and the representativeness heuristic. Social Cognition, 3, 266-279. 